\title{
Application and Research on Carbon Fiber Composite Materials
}

\author{
Huang Yiqiang \\ College of Mechanical Engineering, \\ Linyi University, \\ Linyi, Shandong 276005, China
}

\author{
Li Yunfang* \\ College of Mechanical Engineering, \\ Linyi University, \\ Linyi, Shandong 276005, China \\ *e-mail: liyunfang@lyu.edu.cn
}

\begin{abstract}
This is a review on the structure and performance characteristics of carbon fiber and carbon fiber composite material (CFCM) and their application research progress. Carbon content varies with the type of carbon fiber, and the carbon content is usual more than $90 \%$. Carbon fiber possesses excellent mechanical properties and the specific gravity is a quarter of that of steel. With the constantly improving and perfecting, superior performance of CFCMs has gradually been recognized. This review focuses on the characteristics of carbon fiber and its reinforced composite materials, and the application of CFCM in industrial products and new materials. The further development and application prospects of carbon fiber and its composites are further studied.
\end{abstract}

Keywords-carbon fiber; performance; reinforced composite material; research progress; application prospects

\section{INTRODUCTION}

In the extended area of composite materials, fiber strength materials have been attracted a lot of attention. Since the glass fiber and organic resin composite, the carbon fiber reinforced composite materials have successfully developed, whose performance has greatly improved. Carbon fiber is a kind of special fiber that mainly composed of carbon element, generally in the above $90 \%$. Carbon fiber has the properties of general carbon material, such as resistant to high temperature resistant, friction, conductive, heat conduction and corrosion resistance. However, with general carbon material is different, its appearance has significant anisotropy, soft, can be processed into all kinds of fabric, and along the fiber axis to show high strength. Carbon fiber composite materials (CFCM) are mainly used on military, aerospace, aviation and other sophisticated science and technology fields since 1950s. CFCMs play a great role in in aircraft, rockets, missiles, satellites owing to its high strength and modulus, lightweight, heat resistance, corrosion resistance and other unique performances. Carbon fiber is a kind of special fiber made by carbon element. With the constantly improving and perfecting, superior performance of CFCMs has gradually been recognized. And the substantial drops in price, making their applications in civil industry are gradually expanded. Recently, CFCMs have been widely used in the areas of building construction, textile, petroleum industry, medical machinery and automobile industry.

\section{STRUCTURE AND CHARACTERISTICS OF CFCMS}

Carbon content varies with the type of carbon fiber, and the carbon content is usual more than $90 \%$. Carbon fiber has the characteristics that of general carbon materials, such as high temperature resistance, friction resistance, electrical and thermal conductivity and resistance to corrosion. And carbon fiber is remarkable anisotropic, soft and has high-strength along the fiber axis. Carbon fiber has small specific gravity, so with high specific strength. Carbon fiber is fabricated by artificial chemical fiber which has high carbon content and thermal stability in the heat treatment process. The technology includes the thermal stability and oxidation treatment, carbonization and graphitization processes [1]. Carbon fiber is a new material with excellent mechanical properties and the specific gravity is a quarter of that of steel. The tensile strength of carbon fiber resin composite material is generally above $3500 \mathrm{Mpa}$, which is 7 to 9 times about that of steel, and the tensile elastic modulus is 23000 to $43000 \mathrm{Mpa}$ that is higher than steel. Therefore, the specific strength of CFCMs can reach $2000 \mathrm{Mpa}$ $(\mathrm{g} / \mathrm{cm} 3)$, and that of steel is only $59 \mathrm{Mpa}(\mathrm{g} / \mathrm{cm} 3)$. Carbon fiber can resist the corrosion of common acid and alkali, but has interfacial reaction with metal under high temperature, which damages the fiber seriously [2]. Therefore, to reinforce the metal based composite materials, it should take effective measures to prevent the interfacial reaction. In addition, carbon fiber has good resistance to low temperature, and will not brittle even at the temperature of liquid nitrogen. The advantage of CFCMs is summarized as follows:

1. High strength ( 5 times of steel).

2. Excellent heat resistance (can withstand the temperature higher than $2000^{\circ} \mathrm{C}$ )

3. Excellent thermal shock resistance.

4. Low coefficient of thermal expansion (small deformation).

5. Small heat capacity (energy saving).

6. Small specific weight (a fifth of steel).

7. Excellent resistance to corrosion and radiation.

R. Zhang and his colleagues measured the dynamic tensile moduli of polyethylene-nickel-coated carbon fiber composites with 10 and 4 vol \% NiCF contents under electrical field by a homemade instrument in the frequency range of $100-0.01 \mathrm{~Hz}$ [3]. The drastic descent of the storage modulus of the composite with 10 vol \% was verified in lower frequency range with elevating surface 
temperature by self-heating (Joule heat). The composite was cut when surface temperature was beyond $108^{\circ} \mathrm{C}$. On the other hand, the measurement of the composite with 4 vol \% beyond $88{ }^{\circ} \mathrm{C}$ was impossible, since sufer temperature did not elevate because of the disruption of current networks [3].

\section{THE APPLICATIONS OF CFCMS}

The main application of carbon fiber is to composite with resin, metal, ceramic matrix to fabricate the structural materials. The specific strength and modulus of carbon fiber reinforced epoxy resin composite material is the highest in the existing structural materials. CFCMs possess considerable advantages in the areas that have strict requirements of density, stiffness, weight and fatigue properties, and in the fields that needs high temperature and high chemical stability. Carbon fiber is produced for rocket, aerospace and aviation and other cutting-edge science and technology in 1950s. And now it has been widely used in sports equipment, textile, chemical machinery and medical field. With increasing harsh demanding of cutting-edge technology on the technical performance of the new materials, carbon fibers with high and ultra-high performance are developed, which is another leap in technology, and marks the research and production of carbon fiber has entered a higher stage.

Composite combined by fiber and epoxy resin has become a kind of advanced aerospace materials due to the low density, good rigidity and high strength [4]. When the spacecraft reduce 1 kilogram, the rocket will lose 500 kilograms. In a vertical take-off and landing aircraft, the weight of the used CFCMs has accounted one quarter of the whole machine weight, and one-third of the wing weight. According to reports, the key components of 3 rocket thrusters on the U. S. space shuttle and the launch tube of advanced MX missile are made with advanced CFCMs. Now, most body structures of the first-world racing are made by body structure CFCMs. One major selling point of the world's leading sports car is that almost all the parts are using carbon fibers to enhance the aerodynamics and structural strength. Carbon fiber can also be processed into fabric, blanket, mat, tape, paper and other materials. In addition to be used as insulation materials, carbon fiber mostly used as reinforcing material adding to the resin, metal, ceramic, concrete and other materials to fabricate the composite materials [5-7].

A new approach is to self-weld the short carbon fiber into network throughout the matrix. The SEM images and the platform of storage modulus at higher temperature indicated that carbon fiber owns strong interactions with nylon 6 (PA6), and adding small amount of PA6 leads to the formation of carbon fiber-PA6 networks in the PS matrix. Increasing the contents of PA6 improves the storage modulus at high temperatures, and enhances the strength of the self-welded networks. A larger difference in viscosities between PA6 and the matrix is benefit for increasing NPA6, while a longer hot-press time promotes preferential segregation of PA6 on the carbon fiber surface and further toward the intersections of carbon fiber, and thus greatly improves the mechanical strength of the networks. These CFCMs can storage modulus at $180{ }^{\circ} \mathrm{C}$ increases linearly with encapsulation ratio of PA6, NPA6 [6]. Farther more, carbon fiber reinforced composite materials can be used for structure materials of aircraft and rocket, electromagnetic shielding materials, artificial ligament and other body substitute materials [8].

Recently, a carbon fiber-acrylate system cured by electron beam (EB) was designed to achieve high levels of interfacial adhesion and improved mechanical properties by a reactive sizing [9]. The sizing was made of a partially cured epoxy sizing with a high density of pendant functional groups (acrylate functionality) to facilitate covalent bonding with the matrix. The experiment results showed that the interlaminar shear strength improved from 61 to $81 \mathrm{MPa}(+33 \%)$ without postprocessing, reaching a shear strength similar to that of the same system cured by a thermal treatment [9]. This constitutes a breakthrough for the industrial development of EB curing of composites [9].

Sylvain Giraudet and his group studied the adsorption and electrothermal desorption of volatile organic compounds and siloxanes onto an activated carbon fiber cloth for biogas purification. They investigated the adsorption onto an activated carbon fiber cloth and, in particular, the feasibility of electrothermal desorption [10]. Because incomplete regeneration at $420 \mathrm{~K}$ of two organic compounds (ethanethiol and siloxane D4) from the CFCM from one cycle to another, the mixture of five organic compounds, a constant loss of adsorption capacity was measured after 3 cycles. An average consumption was estimated at $1500 \mathrm{~W} \mathrm{~kg}-1$ of activated carbon fiber cloth [10].

Enrichment of Ventilation Air Methane (VAM) with Carbon Fiber Composites is studied by Jun-Seok Bae and his colleagues [11], which has been an ongoing challenge due to its high volumetric flow rate with low and variable methane concentrations. In their work, honeycomb monolithic carbon fiber composites were developed and employed to capture VAM with a large-scale test unit at various conditions such as VAM concentration, ventilation air (VA) flow rate, temperature, and purging fluids. The results indicated that initial vacuum swing is a control step for the final methane concentration having 5 or 11 times the VAM enrichment by one-step adsorption, which is, the best performance achieved in VAM enrichment technologies worldwide [11].

The carbon fiber and epoxy resin composites have become a kind of advanced aerospace materials owing to their excellent properties. Amanda R. Jones and his group of University of Illinois at Urbana-Champaign have study the autonomic healing of carbon fiber/epoxy interfaces [12]. A maximum of $91 \%$ recovery of interfacial shear strength (IFSS) is achieved for carbon fiber/epoxy interfaces functionalized with capsules containing reactive epoxy resin and ethyl phenyl acetate (EPA). They found a binder is necessary to improve the retention of capsules on the carbon fiber surface. Healing efficiency is assessed by recovery of interfacial shear strength of a single functionalized fiber embedded in a microdroplet of epoxy. The solvent swells the matrix, initiating transport of residual amine functionality from the matrix for further curing with the epoxy resin delivered to the crack plane [12]. In theory, a mechanism of the adhesion between carbon fiber and epoxy resin is studied by using density functional theory (DFT) calculations [13]. This paper constructs two realistic models of adhesion interface consisting of the functionalized carbon surface and a 
fragment of epoxy resin. Calculated values of binding energy are $13.8 \mathrm{kcal} / \mathrm{mol}$ for the $\mathrm{OH}$-functionalized surface and $19.1 \mathrm{kcal} / \mathrm{mol}$ for the $\mathrm{COOH}$-functionalized surface. They strongly suggested that hydrogen bonds between the oxygen-containing functional groups play a crucial role in the adhesive interaction in the carbon fiber/epoxy resin system [13]. Chenggang Chen, etc. prepared carbon fiberreinforced composites used polymer nanocomposite resins. Investigation of the rheological characteristics showed that the modified resin would still be suitable for resin transfer molding of fiber-reinforced composites. The iranocomposite/IM7 carbon fiber composites were uniform with no "filtration" of nanoparticles from the resin during infusion. SAXS results indicate that the nanoclay planes are oriented parallel to the IM7 fiber axis [14].

\section{RESEARCH STATUS OF CFCMS}

In china, carbon fiber has developed for 40 years. Though some achievements have been gained from development to production, the quality and performances do not up to the level of T-300 due to the aspects of raw silk quality, carbonation production technology and equipment, which is seriously restricted the development of aerospace and defense industry business of China. In 2008 June, Jihua Company prepared the carbon fiber that the structure and properties are similar with the T-300 product produced by Japanese Dongli Company. Beijing University of Chemical Technology is now capable of mass producing the carbon fiber with high modulus, and devoting to develop the graphite fiber with high strength and high modulus. And the Shanxi Institute of coal chemistry has been able to industrial produce the carbon fiber products has considerable t level with T-700 products produced by Japanese Dongli Company. Nowadays, the carbon fiber used in aerospace has been developed to the $\mathrm{T}-700, \mathrm{~T}-800$ and even $\mathrm{T}-1000$ series from $\mathrm{T}-300$. However, the technology level, production capacity and product quality of our country still have a large gap with the big industry country of carbon fiber [15].

Nowadays, the production technology of carbon fiber has been improving continuously that the performance is improved rapidly, the cost is gradually reduced and T400-T1000 series of products have been successfully developed. At present, the representative carbon fiber is the large tow polyacrylonitrile based carbon fiber fabricated by America and the small tow carbon fiber produced by Japan. Now the annual production of carbon fiber in the world is about 48000 tons, in which PANbased carbon fiber is the mainstream of development and accounted for more than $90 \%$ of the world carbon fiber market. In recent years, foreign technology research and development of carbon fiber focuses on improving the performance and reducing costs. The Dongli Company of Japanese focuses on the development of carbon fiber with tensile strength of $4000-5000 \mathrm{MPa}$ and comparable price with T-300. Meanwhile, this company also begins to develop large tow carbon fiber, such as T-600S, T-700S and T-700G. In order to meet the large demand for carbon fiber, the manufacturers have to increase the production capacity. The tensile strength and tensile modulus of T1000 typed carbon fiber industrial produced by Dongli Company can reach to $7.02 \mathrm{GPa}$ and $296 \mathrm{GPa}$, respectively. The laboratory has developed the carbon fibers with tensile strength of $9.03 \mathrm{GPa}$, which is $5 \%$ of the theoretical value. Thus, the increase of the tensile strength of carbon fibers has great potential.

\section{CONCLUSION}

The CFCMs is an advanced composite material with high performance and multi-function. Carbon fiber is described as the pinnacle of the comprehensive performance of the material, which is not limited to replace steel material and reinforce epoxy resin composite material due to the highest strength, specific modulus and fiber number. There is no other material has such a series of outstanding performance, so that carbon fiber has irreplaceable advantages in the occasions with high temperature and high chemical stability [16]. At present, the research hotspots about CFCMs home and abroad are focus on the areas that the preparation and the process optimization, the structural damage and bearing capacity analysis of the composite materials. Due to the preparation performance analysis and design of composite material is still relatively backward, there is still a large gap in the CFCMs related areas especially in the aerospace applications compared with developed countries [17].

To expand the application scope of carbon fiber and its composite materials, it should develop the high performance of carbon fiber and break the blockade abroad to meet the demand of the military and aerospace industry. Furthermore, we should develop a new technique to fabricate the carbon fiber with low cost and independent intellectual property rights. It believes that the further development of production and preparation technology of carbon fiber and composite making their applications building, transportation, chemical industry and especial in aerospace, military and high-speed train [18] fields will more widely.

\section{ACKNOWLEDGMENT}

This work is supported by the National Natural Science Foundation of China (Grant No. 51302126), the Natural Science Foundation of Shandong Province, China (Nos. ZR2013EMM001 and ZR2013AL008) and the Startup Foundation for Doctoral Research of Linyi University, Shandong Province, China (No. LYDX2013BS004).

\section{REFERENCES}

[1] Z. H. Sun, Y. Chen, and S. H. Zhou, "Prediction of elasticity of circular woven 3-D tubular carbon fiber composites", Journal of Textile Research, vol. 35, 2014, pp. 56-61.

[2] N. Cene and K. B. Hajrizi, "Behaviour of concrete elements under the shear forces and strengthening with the fibre reinforcemen polymers", Journal of civil engineering and architecture, vol. 8 , 2014, pp. 581-587

[3] R. Zhang, Y. Bin, E. Dong, and M. Matsuo, "Considerable different frequency dependence of dynamic tensile modulus between self-heating (joule heat) and external heating for polymer-nickel-coated carbon fiber composites", J. Phys. Chem. $B$, vol. 118, June 2014, pp. 7047-7058, 10.1021/jp5031202.

[4] C. D. Lan, J. J. Ai, Q. Chen, C. X. Liu, J. P. Wen, and Z. W. Lin. "Study on preparation and properties of epoxy-based shape memory materials reinforced with carbon fibers", Plastics science and technology, vol. 43, 2005, pp. 27-31.

[5] X. Yuan, Y. H.Sun, Y.L. An, and J.H. Zhou, "Preparation and properties of polyphenylene sulfide composite enhanced by CNTs/carbon fibers", Chemistry and adhesion, vol. 0, Tem 2005 , pp.11-14.

[6] Y. H. Zhang and G. H. Wu, "Properties of polymer composites reinforced by self-welded carbon fiber networks", Acta 
polymerica sinica, vol. 0, Jun 2005, pp. 8-14, Doi: 10.11777/j.issn1000-3304.2015.14157.

[7] H. W. Liu, Y. Cheng, and F. Su, "Experimental research on milling forming slot of CFRP", Machine tool \& hydraulics, vol. 42, 2014, pp. 38-41.

[8] X. J. Lv, W. G. Liu, H. Y. Yang, L. Z. Sun, and D. Y. Zhou, "Test validation for pedestrian protection performance of CFRP hood", Automobile technology, vol. 0, 2015, pp. 59-62.

[9] F. Vautard, H. Grappe , and S. Ozcan, "Engineered interface chemistry to improve the strength of carbon fiber composites cured by electron beam", Ind. Eng. Chem. Res., vol. 53, Jul. 2014, pp. 12729-12736, Doi. 10.1021/ie501678j.

[10] S. Giraudet, B.t Boulinguiez, and P. L. Cloirec, “Adsorption and electrothermal desorption of volatile organic compounds and siloxanes onto an activated carbon fiber cloth for biogas purification", Energy Fuels, vol. 28, May 2014, pp. 3924-3932, Doi. 10.1021/ef500600b.

[11] J. S. Bae , S. Su, and X. X. Yu, "Enrichment of Ventilation Air Methane (VAM) with Carbon Fiber Composites", Environ. Sci. Technol., vol. 48,Apr. 2014, pp. 6043-6049, Doi. 10.1021/es500025c.

[12] A. R. Jones, A. Cintora, S. R. White, and N. R. Sottos, "Autonomic healing of carbon fiber/epoxy interfaces", ACS Appl.
Mater. Interfaces, vol. 6, Mar 2014, pp. 6033-6039, Doi. 10.1021/am500536t.

[13] T. Semoto, Y. Tsuji , H. Tanaka , and K. Yoshizawa, "Role of edge oxygen atoms on the adhesive interaction between carbon fiber and epoxy resin", J. Phys. Chem. C, vol. 117,Nov. 2013, pp. 24830-24835, 10.1021/jp407835d.

[14] C. G. Chen, D. Curliss, and B. P. Rice1, "Nanocomposite aerospace resins for carbon fiber-reinforced composites", Defense Applications of Nanomaterials, ACS Symposium Series, Vol. 891, Chapter 7, Jul 2009, pp. 102-118, Chapter Doi: 10.1021/bk2005-0891.ch007.

[15] B. Z. Qian, "Carbon fiber reinforced composite materials: the first choice of aerospace field", Synthetic Fiber in China, vol. 0, 2014, pp. 55-55.

[16] F. Zhang, X. J. Ma, and G. J. Zhao, "Research and Development Status of Wood-based Activated Carbon Fiber", Forestry machinery \& woodworking equipment, vol. 3, 2013, pp. 9-12.

[17] R. Lei, H. fiber reinforced composite materials", Synthetic Fiber in China, vol. 7, 2014, pp. 37-40.

[18] B. Yang, H. J. Liu, and Y. P. Liu, "Prospect of carbon fiber material of high-speed trains", Science and Technology \& Innovation, vol. 0, 2014, pp. 104-105. 